\title{
One-Step Fabrication of Poly(ethylenimine)-Stabilized Silver Nanoparticles from Insoluble Silver Chloride Salt
}

\author{
Kuan Soo Shin* and Ji Hoon Kim \\ Department of Chemistry, Soongsil University, Seoul 156-743, Korea. E-mail: kshin@ssu.ac.kr \\ Received May 11, 2011, Accepted May 27, 2011
}

Key Words : Silver nanoparticles, Silver chloride, Poly(ethylenimine), Insoluble salt

Metal nanoparticles have been intensely investigated in conjunction with their potential use in microelectronics, chemical sensors, data storage, and a host of other applications. $^{1,2}$ The most common synthesis of Ag nanoparticles is the chemical reduction of a silver salt solution by a reducing agent such as $\mathrm{NaBH}_{4}$, citrate, hydrazine, and hydroxylamine hydrochloride. ${ }^{3-5}$ The use of a strong reductant such as borohydride, resulted in small particles that were somewhat monodisperse, but controlling the generation of the larger particles became difficult. The use of citrate, a relatively weaker reductant, resulted in a slower reduction rate, but the size distribution was far from narrow. The controlled synthesis of Ag colloidal nanoparticles was thus attempted using a two-step reduction process to control the particle size. ${ }^{6}$ Furthermore, there is a concern that the usual reducing agents mentioned above may cause environmental toxicity or biological hazards. It has been, therefore, of increasing interest to develop green synthesis of sizecontrolled Ag colloid nanoparticles.

Silver halides, such as $\mathrm{AgCl}$ and $\mathrm{AgBr}$, are water insoluble salts well known for their unusual sensitivity to light. This property has allowed silver halides to become the basis of modern photographic materials. ${ }^{7}$ Silver halides are used to make photographic films since they react with photons to form latent image via photoreduction. As another characteristics, silver halides can, however, react with liquid ammonia to generate a variety of amine complexes. ${ }^{8}$ Most amine complexes derived from silver halides are two-, three-, and, in rare cases, four-coordinate, adopting linear, trigonal planar, and tetrahedral coordination geometries, respectively. ${ }^{8}$

Branched poly(ethylenimine) (PEI), which contains primary, secondary, and tertiary amino groups, has been known to be an efficient agent for the preparation of stabilized $\mathrm{Au}$ nanoparticles. $^{9,10}$ The reductive capability of amines has been known for long time, but the detailed mechanism of how Au nanoparticles are formed by amines has not yet been clarified. In the polyamine-based syntheses of nanoparticles, most of the works have been focused on the preparation of $\mathrm{Au}$ nanoparticles. ${ }^{9-11}$ In contrast, surprisingly, not many works have been published on the synthesis of Ag nanoparticles using polyamine. ${ }^{12,13}$ Herein, we report that the PEI-stabilized Ag nanoparticles can be prepared by reduction of insoluble silver chloride using PEI not only as the dissolving ligand but also as the reductant and the stabilizer. The size and optical properties of PEI-stabilized Ag nanoparticles can be controlled by changing the concentrations of the $\mathrm{AgCl}$ and PEI.

\section{Experimental}

Silver chloride $(\mathrm{AgCl})$ and branched PEI (MW 60 kDa) were purchased from Aldrich, and used as received. Other chemicals, unless specified, were reagent-grade, and highly purified water, with a resistivity greater than $18.0 \mathrm{M} \Omega \cdot \mathrm{cm}$ (Millipore Milli-Q System), was used in preparing aqueous solutions. The PEI-stabilized Ag nanoparticles were prepared by heating an aqueous mixture of $\mathrm{AgCl}$ and $\mathrm{PEI}$ under reflux condition: $0.71 \mathrm{~g}$ of $\mathrm{AgCl}$ was mixed $50 \mathrm{~mL}$ of $0.14 \%(w / w)$ PEI. The PEI-stabilized Ag sol synthesized as such was denoted as Ag-1. Before heating, the $\mathrm{AgCl}$ and PEI mixture was stirred under sonication for $10 \mathrm{~min}$ at room temperature until the mixture became transparent due to the formation of soluble complex between $\mathrm{AgCl}$ and PEI: the turbidity caused by $\mathrm{AgCl}$ gradually disappeared within 10 min. The mixture solution was subsequently heated to 100 ${ }^{\circ} \mathrm{C}$ for $6 \mathrm{~h}$, and then the PEI-stabilized Ag nanoparticles were readily produced. A number of other Ag sols containing Ag nanoparticles of various sizes were obtained by changing the concentrations of reaction mixtures $(\mathrm{AgCl}$ and $\mathrm{PEI})$, and were identified as Ag-2 and Ag-3, as shown in Table 1. The size of the Ag nanoparticles could be controlled by the total

Table 1. Synthesis condition, absorbance peak, and size for Ag nanoparticles

\begin{tabular}{ccccc}
\hline $\begin{array}{c}\mathrm{Ag} \\
\text { Nanoparticles }\end{array}$ & $\begin{array}{c}\text { Amount of } \\
\mathrm{AgCl}\end{array}$ & $\begin{array}{c}\text { Amount of } \\
\text { PEI }\end{array}$ & $\begin{array}{c}\text { Maximum } \\
\text { absorbance }\end{array}$ & $\begin{array}{c}\text { Average } \\
\text { Diameter }\end{array}$ \\
\hline $\mathrm{Ag}-1$ & $0.071 \mathrm{~g}$ & $0.14 \%, 50 \mathrm{~mL}$ & $394 \mathrm{~nm}$ & $8.7 \pm 2.7 \mathrm{~nm}$ \\
$\mathrm{Ag}-2$ & $1.07 \mathrm{~g}$ & $5 \%, 50 \mathrm{~mL}$ & $401 \mathrm{~nm}$ & $23.2 \pm 3.5 \mathrm{~nm}$ \\
$\mathrm{Ag}-3$ & $2.13 \mathrm{~g}$ & $10 \%, 50 \mathrm{~mL}$ & $408 \mathrm{~nm}$ & $30.6 \pm 4.3 \mathrm{~nm}$ \\
\hline
\end{tabular}


amount of PEI and $\mathrm{AgCl}$ added into the reaction mixture. UV-visible (UV-vis) absorption spectra were obtained using an Avantes 3648 spectrometer. Energy-filtering transmission electron microscope (EF-TEM) images were taken on a LIBRA 120 model at $120 \mathrm{kV}$. X-ray diffraction (XRD) patterns were obtained on a Rigaku Model MiniFlex powder diffractometer using $\mathrm{Cu} \mathrm{K}$ radiation. X-ray photoelectron spectroscopy (XPS) measurements were carried out with an AXISHis model using a $\mathrm{Mg} \mathrm{K} \alpha \mathrm{X}$-ray as the light source. Infrared (IR) spectra were measured using a JASCO FT/IR-4100 spectrophotometer.

\section{Results and Discussion}

In this study, an efficient one-step strategy to produce positively charged silver nanoparticles (PEI-stabilized Ag nanoparticles) is developed for the first time, to the best of our knowledge, by the use of polyamine (PEI) and insoluble silver salt $(\mathrm{AgCl})$. Figure 1 shows an increase of the absorbance peak intensity with the reaction time. UV-vis spectra indicate that the absorbance reaches a maximum 4 hour after the addition of PEI to an aqueous $\mathrm{AgCl}$ solution and remains at the same level. Silver nanoparticles often show yellow color, which can be ascribed to the characteristic plasmon absorption of nanosized zero-valent silver crystals. ${ }^{14}$ This clearly reveals that the injected silver chloride was completely dissolved and reduced by PEI molecules and the particle surface was completely covered by PEI. A possible formation mechanism of PEI-stabilized $\mathrm{Ag}$ nanoparticles is a matter of conjecture. As such, the branched PEI polymer was served not only as a dissolving agent, due to complexation of silver cations with amine groups acting as ligands, but also as a reducing template, within which $\mathrm{Ag}$ ions are embedded in PEI polymer, followed by reduction with a reductive capability of amines to form zero valent $\mathrm{Ag}$

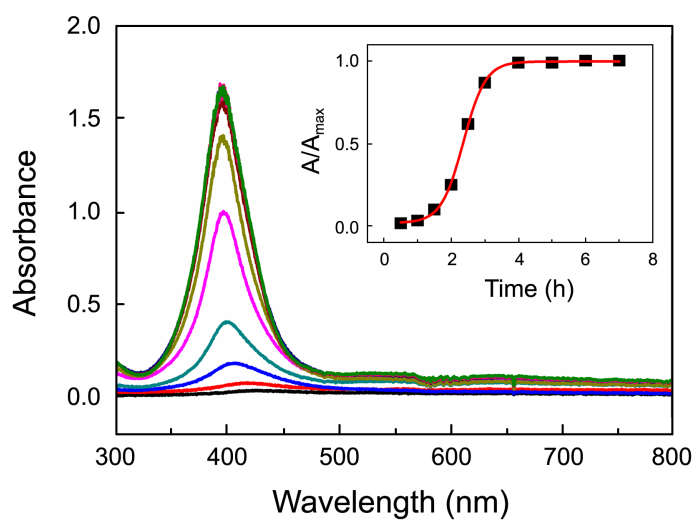

Figure 1. UV-vis spectra obtained as a function of time after the addition of PEI to an aqueous $\mathrm{AgCl}$ solution to prepare $\mathrm{Ag}-1$ nanoparticles. Inset shows the change of absorbance at $\sim 400 \mathrm{~nm}$ as a function of reaction time.

atoms. PEI has also been used to stabilize and control the growth of nanoparticles by agglomerating the Ag atoms, resulting in larger Ag nanoparticles protected by the amine groups of PEI. ${ }^{13}$

The size of the Ag nanoparticles was largely dependent on the concentrations of the PEI and silver salt. By increasing the total concentrations of PEI and $\mathrm{AgCl}$, larger silver particles were produced, and vice versa. Supposedly when the concentrations of both $\mathrm{PEI}$ and $\mathrm{AgCl}$ are increased, more zero valent $\mathrm{Ag}$ atoms are formed by the reductive action of PEI and may be positioned sufficiently enough close to form larger aggregates before they are stabilized by capping with PEI. Approximately 10-30 nm-sized Ag nanoparticles with narrow distributions were prepared, as demonstrated in the EF-TEM images in the upper panels of Figure 2. The particles are spherical in shape. The lower panels of Figure 2 show the size histograms; the average sizes are $8.7 \pm 2.7 \mathrm{~nm}$,
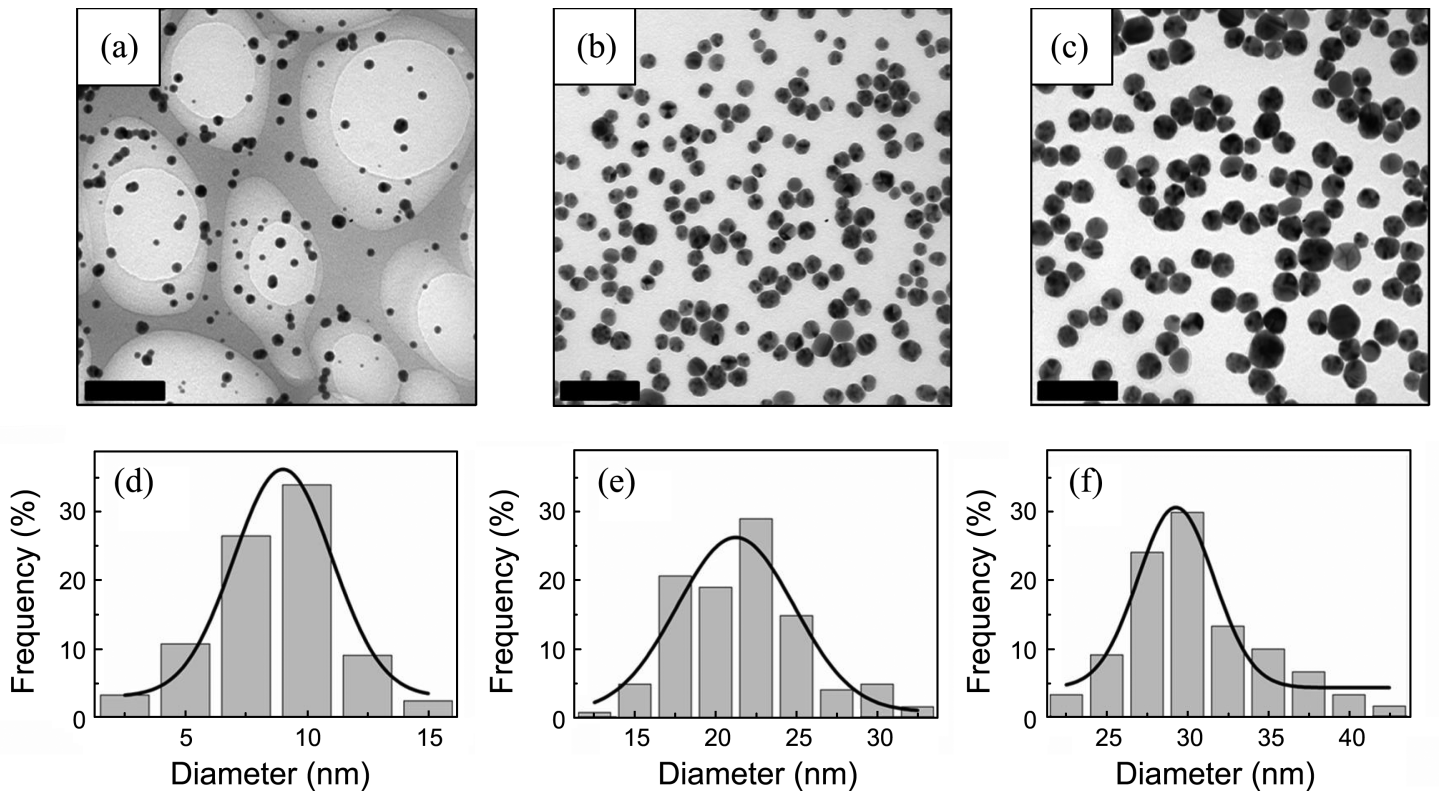

Figure 2. EF-TEM images of (a) Ag-1, (b) Ag-2, and (c) Ag-3 nanoparticles (scale bar = $100 \mu \mathrm{m}$ ). Size histograms of (d) Ag-1, (e) Ag-2, and (f) Ag-3 nanoparticles. 


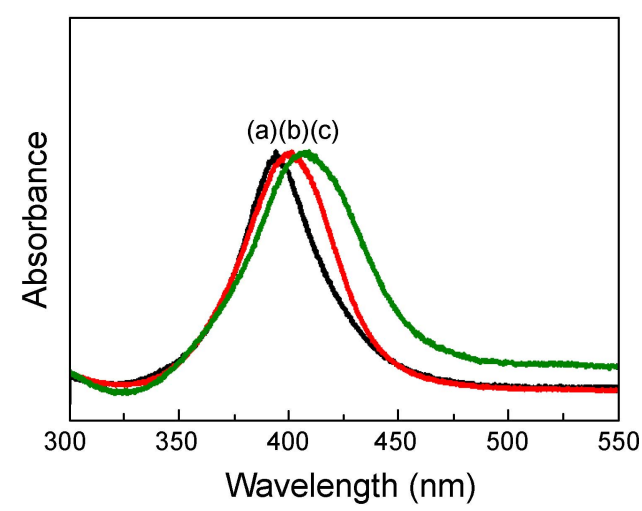

Figure 3. UV-vis spectra of (a) Ag-1, (b) Ag-2, and (c) Ag-3 colloidal solutions.

$23.2 \pm 3.5 \mathrm{~nm}$, or $30.6 \pm 4.3 \mathrm{~nm}$ for Ag-1, Ag-2 and Ag-3 nanoparticles, respectively. Clearly, when the concentrations of PEI and Ag salt are low, smaller particles are formed. In the UV-vis absorption spectra shown in Figure 3, the surface plasmon resonance (SPR) bands of Ag nanoparticles are observed at 394-408 nm, depending on the amount of PEI and $\mathrm{AgCl}$ added into the reaction mixtures. Specifically, the maximum of the SPR bands appears at 394, 401, and 408 $\mathrm{nm}$, respectively, for the Ag sols corresponding to the EFTEM images in Figure 2. The distinct appearance of the SPR bands indicates that the PEI-stabilized Ag nanoparticles are present in a well-dispersed state. The gradual red-shift
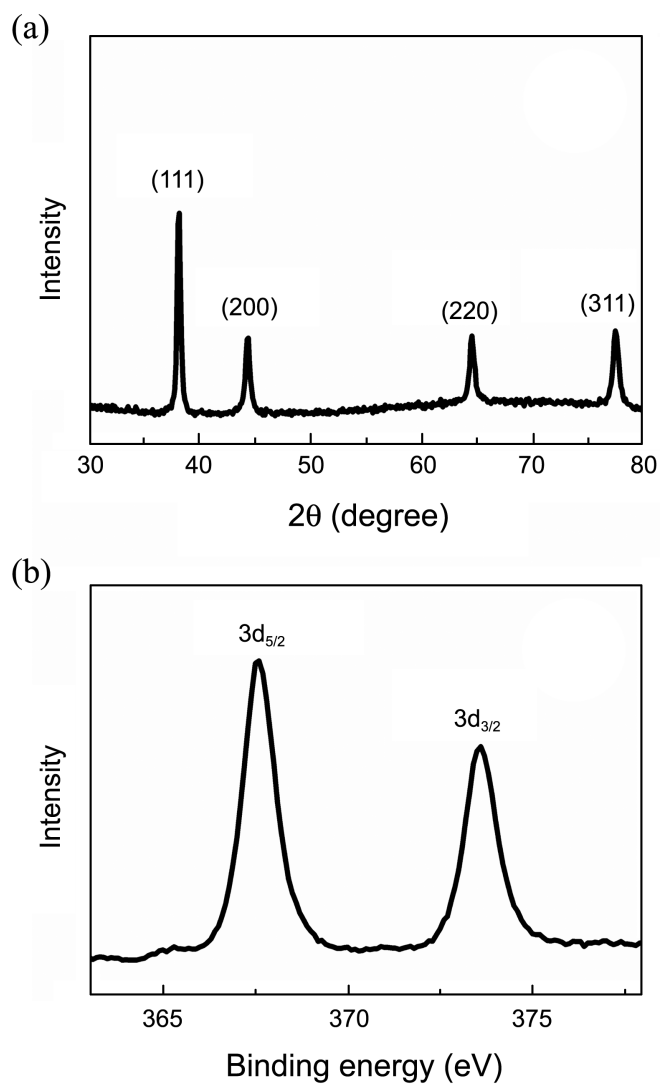

Figure 4. (a) XRD and (b) XPS spectra of PEI-capped Ag-1 nanoparticles.

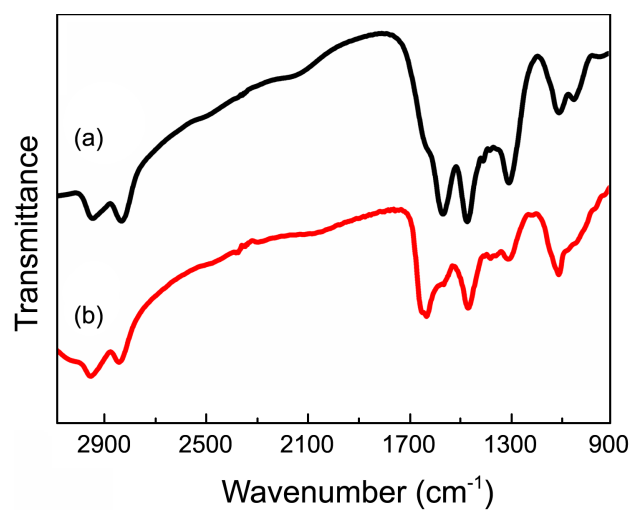

Figure 5. IR spectra of (a) neat PEI and (b) PEI-stabilized Ag-1 nanoparticles.

reflects the production of larger-sized nanoparticles. ${ }^{15}$

The formation of $\mathrm{Ag}$ nanoparticles by the action of PEI can also be confirmed by the XRD pattern (Figure 4(a)) and the XPS spectra (Figure 4(b)). The XRD patterns, which is characterized by the four peaks positioned at $38.2^{\circ}, 44.3^{\circ}$, $64.5^{\circ}$, and $77.4^{\circ}$, in Figure 4(a) can be assigned to the reflections from the (111), (200), (220), and (311) lattice planes, respectively, of the face centered cubic silver particles. ${ }^{16}$ The average size of Ag-1 nanoparticles calculated from Scherrer equation, ${ }^{17}$ using the half width of the intense (111) reflections, was $21 \mathrm{~nm}$, which was larger than the value obtained from EF-TEM images. This is probably because a small number of large particles make a big contribution to the diffraction measurement. To further confirm the formation of $\mathrm{Ag}$ atoms from $\mathrm{AgCl}$, XPS was used to examine the change in oxidation state for $\mathrm{Ag}$ after the reduction reaction had occurred. According to XPS measurements, the XPS peaks at 368 and $374 \mathrm{eV}$ in Figure 4(b) can be assigned, respectively, to the $\mathrm{Ag} 3 \mathrm{~d}_{5 / 2}$ and $\mathrm{Ag} 3 \mathrm{~d}_{3 / 2}$ peaks of zerovalent Ag. We have also measured the IR spectra of neat PEI and PEI-stabilized Ag-1 nanoparticles as shown in Figure 5. The characteristic bands of PEI are associated with the $\mathrm{CH}$ stretching vibrations at $2850-2960 \mathrm{~cm}^{-1}$, the $\mathrm{NH}$ bending vibrations at $1640-1550 \mathrm{~cm}^{-1}$, the $\mathrm{CH}$ bending vibrations at $1500-1300 \mathrm{~cm}^{-1}$, and weak $\mathrm{CN}$ stretching vibrations at 1110$1050 \mathrm{~cm}^{-1} .{ }^{18}$ Based on the IR spectra, we verified the presence of PEI on the surface of Ag nanoparticles. Considering the fact that PEI has long been used as a versatile vector for gene and oligonucleotide transfer into cells, in culture as well as in vivo, ${ }^{19,20}$ PEI-capped Ag nanoparticles are expected to be useful not only for the bio-labeling and cell recognition but also for the purpose of medical diagnostics or acting as gene carriers.

\section{Conclusions}

In this investigation, the size-controlled $\mathrm{Ag}$ nanoparticles were prepared by reduction of insoluble silver chloride using PEI. The PEI polymer was served not only as a dissolving agent but also as a reducing agent to form zero valent $\mathrm{Ag}$ atoms. PEI was also used to stabilize and control the growth 
of nanoparticles by agglomerating the Ag atoms, resulting in Ag nanoparticles protected by the amine groups of PEI. The size and optical properties of PEI-stabilized Ag nanoparticles could be controlled by changing the concentrations of the PEI and $\mathrm{AgCl}$. By increasing the total concentrations of PEI and $\mathrm{AgCl}$, larger silver particles were produced, and vice versa: the $\mathrm{Ag}$ nanoparticles synthesized ranged in size from 10 to $30 \mathrm{~nm}$ with narrow size distributions.

Acknowledgments. This work was supported by the Soongsil University Research Fund.

\section{References}

1. Link, S.; El-Sayed, M. A. Annu. Rev. Phys. Chem. 2003, 54, 331.

2. Polavarapu, L.; Venkatram, N.; Ji, W.; Xu, Q. H. Appl. mater. Interfaces 2009, 1, 2298.

3. Lee, P. C.; Meisel, D. J. Phys. Chem. 1982, 86, 3391.

4. Nickel, U.; Mansyreff, K.; Schneider, S. J. Raman Spectrosc. 2004, 35, 101.

5. Leopold, N.; Lendl, B. J. Phys. Chem. B 2003, 107, 5723.
6. Shirtcliffe, N.; Nickel, U.; Schneider, S. J. Colloid Interface Sci. 1999, 211, 122 .

7. Greenwood, N. N.; Earnshaw, A. Chemistry of the Elements Permagon Press: New York, 1984; 1185.

8. Leden, I.; Persson, G. Acta Chem. Scand. 1961, 15, 607.

9. Kim, K.; Lee, H. B.; Lee, J. W.; Park, H. K.; Shin, K. S. Langmuir 2008, 24, 7178.

10. Sun, X.; Dong, S.; Wang, E. Mater. Chem. Phys. 2006, 96, 29.

11. Sun, X.; Dong, S.; Wang, E. Langmuir 2005, 21, 4710.

12. Sun, X.; Dong, S.; Wang, E. Macromolecules 2004, 37, 7105.

13. Kim, K.; Lee, H. B.; Lee, J. W.; Shin, K. S. J. Colloid Interface Sci. 2010, 345, 103.

14. Mulvaney, P. Langmuir 1996, $12,788$.

15. Sun, X.; Luo, Y. Material Letters 2005, 59, 3847.

16. Liu, C.; Yang, X.; Yuan, H.; Zhou, Z.; Xiao, D. Sensors 2007, 7, 708.

17. Birks, L. S.; Friedman, H. J. Appl. Phys. 1946, 17, 687.

18. Kisner, A.; Lenk, S.; Mayer, D.; Mourzina, Y.; Offenhäusser, A. J. Phys. Chem. C 2009, 113, 20143.

19. Godbey, W. T.; Wu, K. K.; Mikos, A. G. Medical Science 1999, 96, 5177.

20. Boussif, O.; Lezoualc'h, F.; Zenta, M. A.; Mergny, M. D.; Scherman, D.; Demeneix, B.; Behr, J. P. Proc. Natl. Acad. Sci. 1995, 92, 7297. 